\title{
Dynamic Network Security Deployment Under Partial Information
}

\author{
(Invited Paper) \\ George Theodorakopoulos \\ EPFL \\ Lausanne, Switzerland \\ Email: george.theodorakopoulos@epfl.ch \\ John S. Baras \\ University of Maryland \\ College Park, MD, USA \\ Email: baras@umd.edu
}

\author{
Jean-Yves Le Boudec \\ EPFL \\ Lausanne, Switzerland \\ Email: jean-yves.leboudec @ epfl.ch
}

\begin{abstract}
A network user's decision to start and continue using security products is based on economic considerations. The cost of a security compromise (e.g., worm infection) is compared against the cost of deploying and maintaining a sufficient level of security. These costs are not necessarily the real ones, but rather the perceived costs, which depend on the amount of information available to a user at each time. Moreover, the costs (whether real or perceived) depend on the decisions of other users, too: The probability of a user getting infected depends on the security deployed by all the other users.

In this paper, we combine an epidemic model for malware propagation in a network with a game theoretic model of the users' decisions to deploy security or not. Users can dynamically change their decision in order to maximize their currently perceived utility. We study the equilibrium points, and their dependence on the speed of the learning process through which the users learn the state of the network. We find that the faster the learning process, the higher the total network cost.
\end{abstract}

\section{INTRODUCTION}

Mobile devices (cellphones, smartphones, pocket PCs, etc.) acquire more and more capabilities in terms of both computation and communication. Processors are getting faster, memory and storage capacity increase, and as a consequence applications for such devices multiply in number and complexity. On the other hand, communication interfaces multiply, too: Cellphones used to have only GSM protocol implementations, but now most have also GPRS, UMTS, Bluetooth, and of course many smartphones are WiFi enabled. These capabilities have made mobile devices very popular. Their popularity combined with their capabilities have made them an attractive target for malware [1], [2].

No user likes his device to be infected with malware; on the other hand, installing and using security software costs money, battery, CPU resources, and usability. Therefore, users have to make a tradeoff between staying unprotected (thus risking infection) and buying protection (thus incurring protection costs). To make an informed tradeoff, the users need to estimate the risk of infection, for which they need to know the size of existing infections (if any). The more current the infection information is, the better the cost optimization that the users can do.

A mobile operator can warn users for currently ongoing infections. Should it? How frequently? The operator wants to reduce the total network cost, but the users want to reduce their personal expected cost. Are these two objectives aligned?

These questions have not been adequately addressed. Traditionally, research has focused on modeling only the propagation of worms, in the Internet [3], [4], [5], and also in mobile networks [6], [7], [8], among others. Research that goes beyond modeling propagation to model countermeasures has not explicitly taken into account the users' freedom to install protection or not. The decision to deploy countermeasures was assumed to be taken unilaterally by the operator (e.g., [9]) or, at any rate, always accepted by the users (e.g., [10], [11]). The work most similar to ours [12] has only modeled static decisions, where users decide once and for all. At the same time, users are assumed to have perfect knowledge of all relevant parameters, and also perfect reasoning capabilities.

The main modeling novelty in the present work is the combination of a worm propagation model with a game theoretic process (which involves learning) that determines users' actions: The users maximize their perceived personal cost through their decision to install or uninstall protection and this decision depends on the information that the users possess about the state of the network. That information is given to them at some update rate from the operator. Consequently, it is not current at all times, since the network state changes, first, because of the propagation of the worm, and, second, because of the decisions of other users. Also, our users do not take into account the effect that their actions will have on the network state, and therefore on the future actions of other users. In that, they have limited reasoning capabilities.

We find game theoretic equilibria, which are stable in the sense that if a small proportion of the population changes strategy, or if a small proportion of infected users enters the population, then the dynamics of the system lead it back to the stable equilibrium point. Our main finding is that the total cost to the network increases as the update rate increases.

The rest of the paper is organized as follows: In Section II, we describe the system model, the two components of which are the epidemic worm propagation, and the game theoretic decision making procedure. Section III presents the results about the equilibrium points and their stability, and 
Section IV concludes with some practical interpretation of our results. More detailed derivations of the results are in the Appendix.

\section{SyStem Model}

\begin{tabular}{|c|l|}
\hline \multicolumn{2}{|c|}{ List of Variables } \\
\hline$\beta$ & contact rate per user per time unit \\
$\gamma$ & update rate (rate of learning from the monitor) \\
$\delta$ & rate of recovery from the infection \\
$N$ & total number of users $(N \rightarrow \infty)$ \\
$S$ & fraction of Susceptible users in the network \\
$I$ & fraction of Infected users in the network \\
$P$ & fraction of Protected users in the network \\
$c_{I}$ & cost of infection \\
$c_{P}$ & cost of protection \\
$p_{S P}(I)$ & Probability that a Susceptible user switches \\
$p_{P S}(I)$ & to Protected when learning $I$ (see Eq. $(3))$ \\
& Probability that a Protected user switches \\
& to Susceptible when learning $I$ \\
$\epsilon$ & (p $\left.p_{P S}(I)=1-p_{S P}(I)\right)$ \\
$I^{*}$ & shape parameter of $p_{S P}(I)$ \\
& position parameter of $p_{S P}(I),\left(I^{*}=\frac{c_{P}}{c_{I}}<1\right)$ \\
\hline \multicolumn{2}{|c}{ TABLE I } \\
& $\quad$ LIST of VARIABLES
\end{tabular}

\section{A. Epidemic-based Worm Propagation Model}

We assume a network with $N$ users, and the total contact rate is $\beta N$ contacts per time unit in the whole network, where $\beta>0$ is constant with respect to $N$. So, a given user makes $\beta$ contacts per time unit, and a given pair makes $\frac{2 \beta}{N-1}$ contacts per time unit. Each user can be at each time instant $t$ in one of three states: Susceptible (S), Infected (I), Protected (P). We denote $S(t), I(t), P(t)$ the respective percentages $(S(t)+I(t)+P(t)=1$; the time dependence will not be explicitly given in the rest of the paper). A worm propagates in the network, infecting susceptible users. The infection lasts for a random amount of time, exponentially distributed with parameter $\delta>0$. While being infected, a user infects other susceptible users he contacts. After the infection is over, the user becomes protected.

For the worm behavior described above, we use the standard SIR model [13], which we will call SIP ( $\mathrm{P}$ for Protected):

$$
\begin{aligned}
\frac{d}{d t} S & =-2 \beta S I \\
\frac{d}{d t} I & =2 \beta S I-\delta I \\
\frac{d}{d t} P & =\delta I
\end{aligned}
$$

\section{B. Game Theoretic Decision Making}

The non-infected users ( $\mathrm{S}$ and $\mathrm{P}$ ) can choose whether to remain in their current state or switch to the other state. Their choice depends on the risk of getting infected versus the cost of protection. The cost of protection is fixed at $-c_{P}<0$, but the risk of getting infected depends on the infection cost $-c_{I}<-c_{P}<0$ and also on the percentage $I$ of infected users in the network. We define the total network cost to be the quantity

$$
C(I, P)=c_{I} I+c_{P} P .
$$

We encode these considerations in the following game description. There are two player types: Type 1 is noninfected, and Type 2 is infected. We are interested in the viewpoint of a Type 1 player. The players are matched at random, so the probability that a given Type 1 player will meet a Type 2 player is equal to the percentage of the infected users $I$.

The Type 1 versus Type 1 player game is:

\begin{tabular}{|c|c|c|}
\hline & $\mathrm{S}$ & $\mathrm{P}$ \\
\hline $\mathrm{S}$ & $(0,0)$ & $\left(0,-c_{P}\right)$ \\
\hline $\mathrm{P}$ & $\left(-c_{P}, 0\right)$ & $\left(-c_{P},-c_{P}\right)$ \\
\hline
\end{tabular}

and the Type 1 versus Type 2 player game is:

\begin{tabular}{|l|c|}
\hline & $\mathrm{I}$ \\
\hline $\mathrm{S}$ & $\left(-c_{I},-\right)$ \\
\hline $\mathrm{P}$ & $\left(-c_{P},-\right)$ \\
\hline
\end{tabular}

where we omit the payoffs of the Type 2 (infected) player.

If the non-infected players know the fraction $I$, they can choose their best response as follows: Choose $\mathrm{S}$ if $-c_{I} I>$ $-c_{P} \Rightarrow I<\frac{c_{P}}{c_{I}} \equiv I^{*}$, and $\mathrm{P}$ otherwise.

The users need to learn the value of $I$. For the learning process, we assume there is a centralized service for monitoring the network, which knows instantly the current percentage of infected users in the network. The users contact the service at rate $\gamma$ times per time unit, and learn the value of $I$.

As a consequence, the users do not know the exact value of $I$ at all times. More importantly, it has been observed [14, Ch. 4] that when asked to choose between two alternatives, behavior is random, becoming more deterministic when the two alternatives become more distinct. In our case, "more distinct" translates to larger utility difference. To model this random aspect of the users' behavior, we assume that a user switches from $\mathrm{S}$ to $\mathrm{P}$ with probability $p_{S P}(I)$, and from $\mathrm{P}$ to $\mathrm{S}$ with probability $p_{P S}(I)$. We set $p_{S P}(I)$ to be the following piecewise linear function (see Fig. 1):

$$
p_{S P}(I)= \begin{cases}0 & I<I^{*}-\frac{\epsilon}{2} \\ \frac{1}{\epsilon}\left(I-I^{*}+\frac{\epsilon}{2}\right) & I^{*}-\frac{\epsilon}{2}<I<I^{*}+\frac{\epsilon}{2} \\ 1 & I>I^{*}+\frac{\epsilon}{2}\end{cases}
$$

and $p_{P S}(I)=1-p_{S P}(I)$. For $\epsilon \rightarrow 0$, this function becomes a pure best response function. For $\epsilon>0$ it is a smoothed best response. The best response and smoothed best response dynamics are common models for the learning behavior of game theoretic users [14, Ch. 3 and 4].

\section{Learning Through Centralized Monitoring: The Complete Model}

The users learn the percentage $I$ of infected users at rate $\gamma$, the update rate, which leads to the following system:

$$
\begin{aligned}
\frac{d}{d t} S & =-2 \beta S I-\gamma S p_{S P}(I)+\gamma P p_{P S}(I) \\
\frac{d}{d t} I & =2 \beta S I-\delta I \\
\frac{d}{d t} P & =\delta I+\gamma S p_{S P}(I)-\gamma P p_{P S}(I)
\end{aligned}
$$




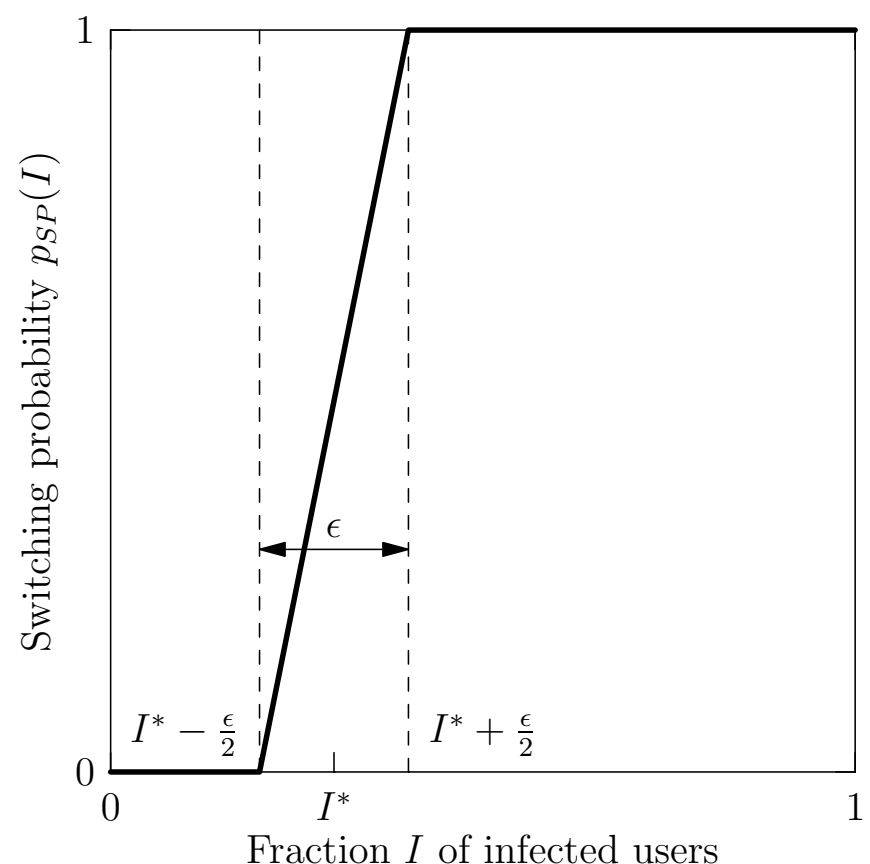

Fig. 1. The probability that a Susceptible user switches to being Protected, when learning the fraction $I$ of Infected users in the network.

Note that one of the three equations is redundant, since we know that $S+I+P=1$ at all times. We keep the first two, together with $S+I+P=1$, from which we substitute $P$ into the first one.

\section{Mathematical Models}

Our model (i.e., the system (4)) describes an evolutionary game on the simplex in $\mathbb{R}^{3}$, since $S+I+P=1$ [15]. Such games on the simplex have been intensively studied over the last twenty years as they are very relevant for biological and social population dynamics, e.g., replication dynamics [15], [16], [17], [18], [19], [20], [21]. More specifically, it is possible to study the dynamics and equilibria of our model globally using the methods of [15], [16], [17], [18], [19], [20], [21], which originated in the seminal work of Shahshahani [15]. The methods involved include Lie-algebraic conditions, potential games and Lyapunov functions.

It is also possible to view our model as a switching system (see [22], [23], [24], as examples) on the simplex in $\mathbb{R}^{3}$, where the switching controls are the probabilities, $p_{S P}($. and $p_{P S}($.$) ; actually only one is needed. Then one can$ formulate more general dynamic games where these controls can depend on the past history of $I(t)$ in various ways. Other formulations can be also studied where the whole problem is considered in either finite or infinite time, as a stochastic game with switching strategies, still on the simplex in $\mathbb{R}^{3}$.

All these more general frameworks and formulations are considered in our forthcoming paper [25].

\section{Results: Equilibrium Points And Stability}

Our main finding is

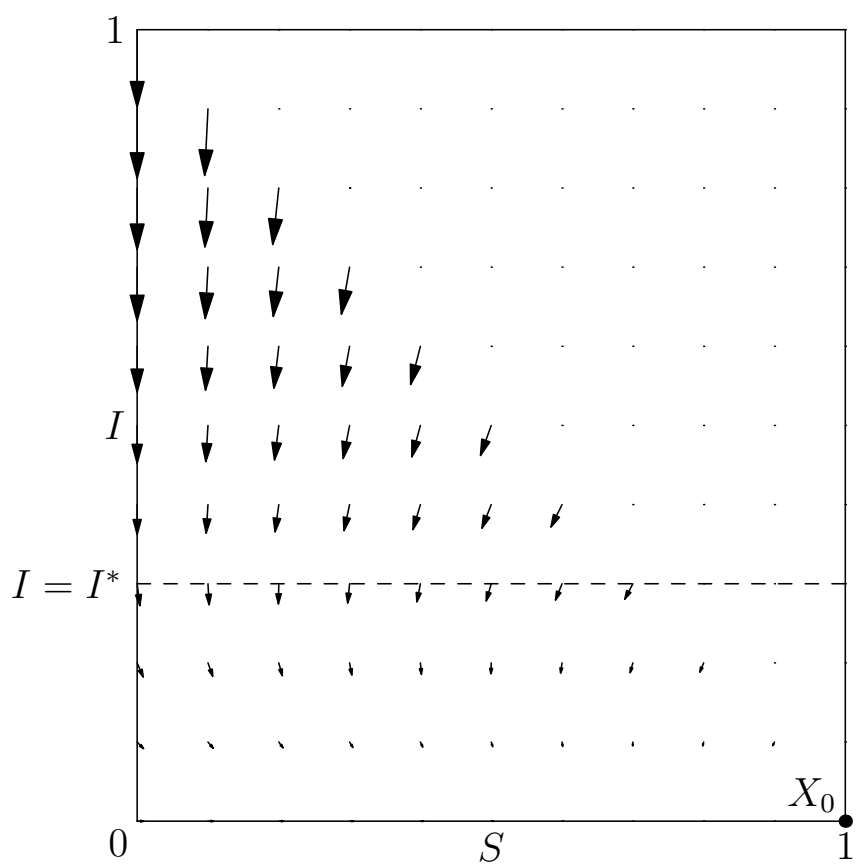

Fig. 2. The vector field of the system (4) for the case $\delta>2 \beta$. At the point $(S, I)$, an arrow parallel to $\left(\frac{d S}{d t}, \frac{d I}{d t}\right)$ is plotted. The only equilibrium point is $X_{0}=(1,0)$. It is also stable.

Theorem 1. In the stable equilibrium points of our system, the total network cost $C(I, P)$ monotonically decreases with the update rate $\gamma$. So, the value of $\gamma$ that minimizes $C(I, P)$ is $\gamma_{\text {opt }}=0$.

In this section we state our findings about the equilibrium points and their stability. The derivations are in the Appendix.

We look for equilibrium points by solving the system:

$$
\begin{aligned}
& 0=-2 \beta S I-\gamma S p_{S P}(I)+\gamma P p_{P S}(I) \\
& 0=2 \beta S I-\delta I \\
& 1=S+I+P
\end{aligned}
$$

If $\delta>2 \beta$, the only equilibrium point is

$$
X_{0}=\left(S_{0}, I_{0}, P_{0}\right)=(1,0,0)
$$

and it is stable. The vector field of the system (4) corresponding to the condition $\delta>2 \beta$ is shown in Fig. 2

If $\delta<2 \beta$, the point $X_{0}$ is still an equilibrium point but it is no longer stable. Now, there are two new potential equilibrium points:

$$
X_{1}=\left(S_{1}, I_{1}, P_{1}\right)=\left(\frac{\delta}{2 \beta}, \frac{1-\frac{\delta}{2 \beta}}{1+\frac{\delta}{\gamma}}, \frac{1-\frac{\delta}{2 \beta}}{1+\frac{\gamma}{\delta}}\right)
$$

and

$$
X_{2}=\left(S_{2}, I_{2}, P_{2}\right)=\left(\frac{\delta}{2 \beta}, I_{2}, 1-\frac{\delta}{2 \beta}-I_{2}\right),
$$

where $I_{2}$ is the smallest solution of the equation

$$
I^{2}-\left(\frac{\epsilon}{2}+\frac{\delta \epsilon}{\gamma}+1+I^{*}\right) I+\epsilon\left(1-\frac{\delta}{2 \beta}\right)+I^{*}-\frac{\epsilon}{2}=0 .
$$




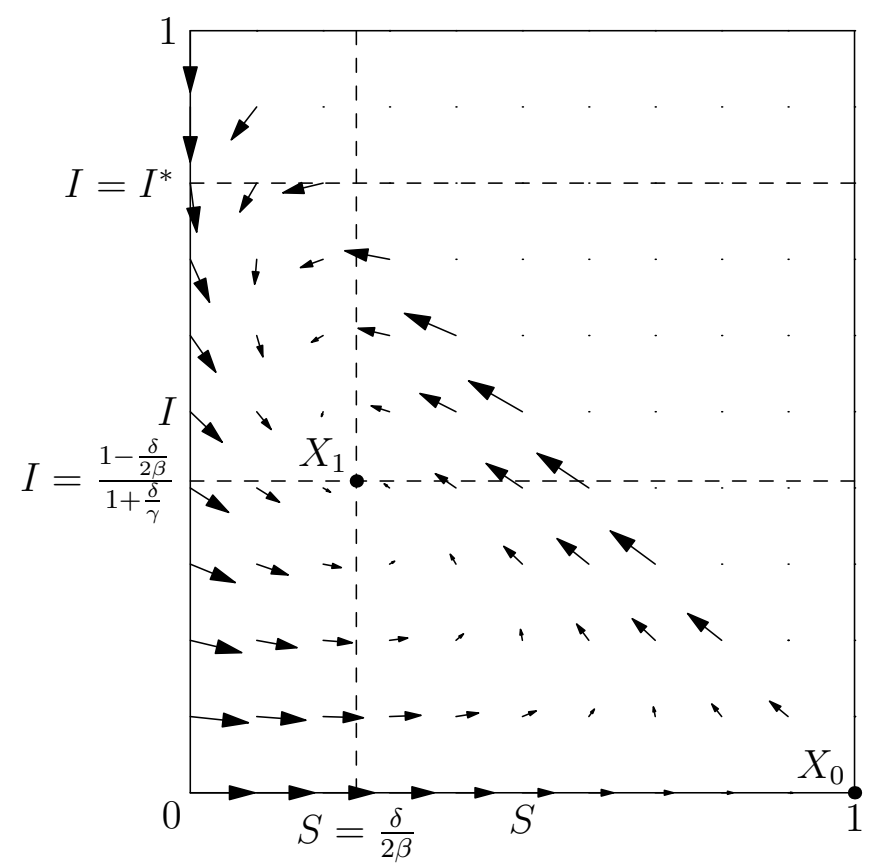

Fig. 3. The vector field of the system (4) for the case $\delta<2 \beta$ and $\frac{1-\frac{\delta}{2 \beta}}{1+\frac{\delta}{\gamma}}<I^{*}-\frac{\epsilon}{2}$. At the point $(S, I)$, an arrow parallel to $\left(\frac{d S}{d t}, \frac{d I}{d t}\right)$ is plotted. The point $X_{1}=\left(\frac{\delta}{2 \beta}, \frac{1-\frac{\delta}{2 \beta}}{1+\frac{\delta}{\gamma}}, \frac{1-\frac{\delta}{2 \beta}}{1+\frac{\gamma}{\delta}}\right)$ is an equilibrium point and it is stable. The point $X_{0}=(1,0)^{\gamma}$ is also an equilibrium point but it is unstable. We can see that all trajectories converge to $X_{1}$, except those that start on the axis $I=0$, which converge to $X_{0}$.

That is,

$$
\begin{aligned}
I_{2}= & \frac{1}{2}\left(\frac{\epsilon}{2}+\frac{\delta \epsilon}{\gamma}+1+I^{*}-\right. \\
& \left.\sqrt{\left(\frac{\epsilon}{2}+\frac{\delta \epsilon}{\gamma}+1+I^{*}\right)^{2}-4\left(\epsilon\left(\frac{1}{2}-\frac{\delta}{2 \beta}\right)+I^{*}\right)}\right) .
\end{aligned}
$$

The point $X_{1}$ exists and is stable if and only if (in addition to $\delta<2 \beta$ )

$$
\frac{1-\frac{\delta}{2 \beta}}{1+\frac{\delta}{\gamma}}<I^{*}-\frac{\epsilon}{2}
$$

Otherwise, the point $X_{2}$ exists and is stable. Figures 3 and 4 show the vector fields of the system (4) in the two cases where $X_{1}$ and $X_{2}$, respectively, exist and are stable.

The stability in all cases was checked through the evaluation of the Jacobian at each point. In all cases, the Jacobian had eigenvalues with negative real part. In the case of $X_{1}$ and $X_{2}$, there can be an imaginary part for some values of the parameters, so we have diminishing oscillations (spiral). In particular, we have a spiral around $X_{1}$ when $\beta$ is close enough to $\gamma\left(1+\frac{\gamma}{\delta}\right)^{2}-\frac{\gamma}{2}$, and around $X_{2}$ when $\epsilon$ is small enough.

Note that the evaluation of the Jacobian proves local stability (i.e., if the perturbation from the equilibrium point is small enough, the system returns to it). However, the

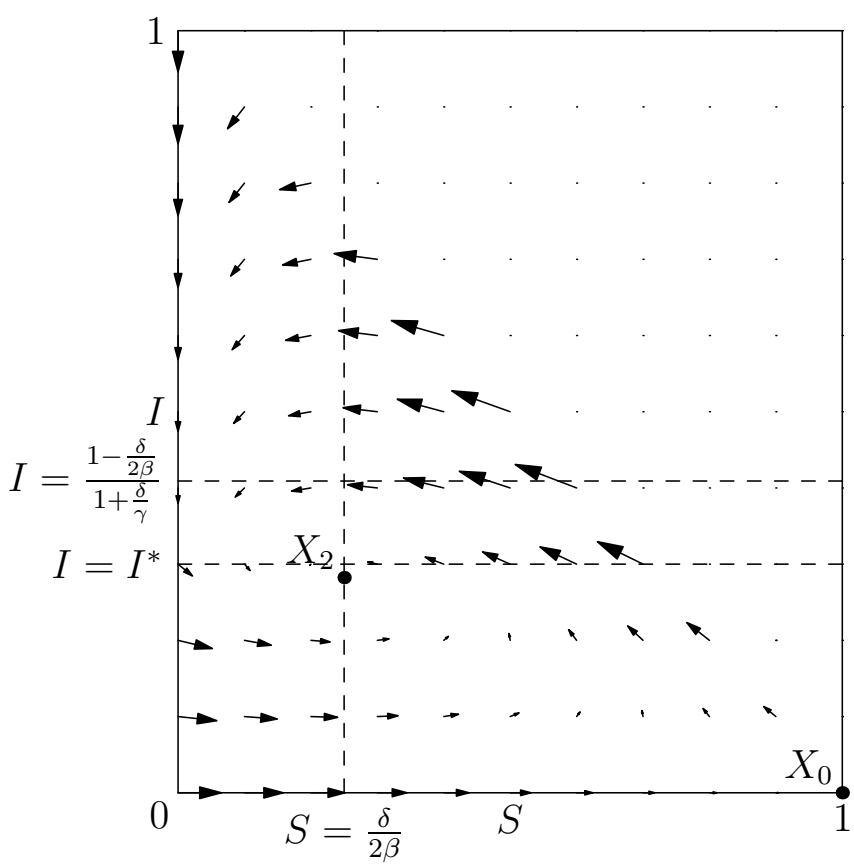

Fig. 4. The vector field of the system (4) for the case $\delta<2 \beta$ and $\frac{1-\frac{\delta}{2 \beta}}{1+\frac{\delta}{\gamma}}>$ $I^{*}-\frac{\epsilon}{2}$. At the point $(S, I)$, an arrow parallel to $\left(\frac{d S}{d t}, \frac{d I}{d t}\right)$ is plotted. The point $X_{2}=\left(\frac{\delta}{2 \beta}, I_{2}, 1-\frac{\delta}{2 \beta}-I_{2}\right)$ (for the value of $I_{2}$ see (10)) is a stable equilibrium point, always within $\frac{\epsilon}{2}$ below $I^{*}$ (see Appendix). The point $X_{0}=(1,0)$ is also an equilibrium point but it is unstable. We can see that all trajectories converge to $X_{2}$, except those that start on the axis $I=0$, which converge to $X_{0}$.

figures imply global stability, that is, the system returns to the (unique for each combination of parameters) regardless of the initial conditions, or the size of the perturbation away from the equilibrium point. The only exception is when the system starts on the $I=0$ axis, in which case it converges to the unstable point $X_{0}$. We indeed investigate and establish global stability results for this problem in [25].

We can now prove Theorem 1 (that network cost in the equilibrium points $X_{0}, X_{1}, X_{2}$ increases with $\gamma$ ) by differentiating $I_{1}$ and $I_{2}$ with respect to $\gamma$. At the point $X_{0}$, the value $I_{0}=0$ does not depend on $\gamma$, and so the network cost in that case is 0 for all values of $\gamma$.

$$
\begin{aligned}
\frac{d I_{1}}{d \gamma} & =\frac{d}{d \gamma} \frac{1-\frac{\delta}{2 \beta}}{1+\frac{\delta}{\gamma}} \\
\Rightarrow & \frac{d I_{1}}{d \gamma}=\left(1-\frac{\delta}{2 \beta}\right) \frac{\frac{\delta}{\gamma^{2}}}{\left(1+\frac{\delta}{\gamma}\right)^{2}} \\
\Rightarrow & \frac{d I_{1}}{d \gamma}>0, \forall \gamma
\end{aligned}
$$

To find $\frac{d I_{2}}{d \gamma}$ we differentiate (9), which $I_{2}$ satisfies, with respect to $\gamma$ :

$I_{2}^{2}-\left(\frac{\epsilon}{2}+\frac{\delta \epsilon}{\gamma}+1+I^{*}\right) I_{2}+\epsilon\left(1-\frac{\delta}{2 \beta}\right)+I^{*}-\frac{\epsilon}{2}=0$ 


$$
\begin{aligned}
& \Rightarrow 2 I_{2} \frac{d I_{2}}{d \gamma}+I_{2} \frac{\delta \epsilon}{\gamma^{2}}-\left(\frac{\epsilon}{2}+\frac{\delta \epsilon}{\gamma}+1+I^{*}\right) \frac{d I_{2}}{d \gamma}=0 \\
& \Rightarrow\left(2 I_{2}-\left(\frac{\epsilon}{2}+\frac{\delta \epsilon}{\gamma}+1+I^{*}\right)\right) \frac{d I_{2}}{d \gamma}+\frac{\delta \epsilon}{\gamma^{2}} I_{2}=0
\end{aligned}
$$

We know that $I_{2}<1$ and $I_{2}<I^{*}+\frac{\epsilon}{2}$. So, $2 I_{2}-\left(\frac{\epsilon}{2}+\right.$ $\left.\frac{\delta \epsilon}{\gamma}+1+I^{*}\right)$ is always negative. It follows that

$$
\frac{d I_{2}}{d \gamma}>0, \forall \gamma
$$

From (12) and (14) we conclude that $\frac{d I_{2}}{d \gamma}>0, \forall \gamma$. Recalling that $S_{1}=S_{2}=\frac{\delta}{2 \beta}$, we can see that the total network cost increases with $\gamma$, proving Theorem 1:

$$
\begin{aligned}
C(I, P) & =c_{I} I+c_{P} P \\
& =c_{I} I+c_{P}\left(1-\frac{\delta}{2 \beta}-I\right) \\
& =\left(c_{I}-c_{P}\right) I+c_{P}\left(1-\frac{\delta}{2 \beta}\right) \\
\Rightarrow \frac{d C(I, P)}{d \gamma} & =\left(c_{I}-c_{P}\right) \frac{d I}{d \gamma} \\
\Rightarrow \frac{d C(I, P)}{d \gamma} & >0, \forall \gamma
\end{aligned}
$$

\section{CONCLUSiON}

The users' ability to switch from $\mathrm{P}$ to $\mathrm{S}$, and vice versa, leads to a sustained infection level (endemic), if $\frac{\delta}{2 \beta}<1$. The actual fraction of the I at the equilibrium point (which is proportional to the total network cost) increases monotonically with the update rate $\gamma$. This counterintuitive conclusion is an instance of the price of anarchy phenomenon: The individually rational outcome (large $\gamma$ implies more current information available to the users) is worse than the socially optimal outcome.

The conflict between network optimality and individual optimality creates a dilemma for the operator. Not notifying the users might be interpreted as trying to manipulate them into buying unnecessary protection. Acquiescing to their demands and updating them as quickly as possible would be both expensive in terms of, e.g., monitoring infrastructure, and total network cost, as we have proven. Therefore, a sensible compromise seems to be for the operator to shoulder at least part of the protection costs, perhaps as part of the package offered to users.

\section{ACKNOWLEDGMENT}

The research of the second author was supported by the Communications and Networks Consortium sponsored by the U.S. Army Research Laboratory under the Collaborative Technology Alliance Program, Cooperative Agreement DAAD19-01-2-0011, by the U.S. Army Research Office under MURI award W 911 NF 0710287, and by the MAST Consortium sponsored by the U.S. Army Research Laboratory under the Collaborative Technology Alliance Program, Cooperative Agreement W911NF-08-2-0004.

\section{REFERENCES}

[1] M. Hypponen, "Malware goes mobile," Scientific American, pp. 70-77, November 2006.

[2] J. Kleinberg, "Computing: The wireless epidemic," Nature, vol. 449, no. 7160 , pp. 287-288, September 2007, biological connection.

[3] J. Kephart and S. White, "Directed-graph epidemiological models of computer viruses," Research in Security and Privacy, 1991. Proceedings., 1991 IEEE Computer Society Symposium on, pp. 343-359, 20-22 May 1991.

[4] J. O. Kephart and S. R. White, "Measuring and modeling computer virus prevalence," in SP '93: Proceedings of the 1993 IEEE Symposium on Security and Privacy. Washington, DC, USA: IEEE Computer Society, 1993, p. 2.

[5] C. C. Zou, W. Gong, and D. Towsley, "Code red worm propagation modeling and analysis," in CCS '02: Proceedings of the 9th ACM conference on Computer and communications security. New York, NY, USA: ACM, 2002, pp. 138-147.

[6] C. Fleizach, M. Liljenstam, P. Johansson, G. M. Voelker, and A. Mehes, "Can you infect me now?: malware propagation in mobile phone networks," in WORM '07: Proceedings of the 2007 ACM workshop on Recurring malcode. New York, NY, USA: ACM, 2007, pp. 6168.

[7] H. Zheng, D. Li, and Z. Gao, "An epidemic model of mobile phone virus," Pervasive Computing and Applications, 2006 1st International Symposium on, pp. 1-5, 3-5 Aug. 2006.

[8] I. Carreras, D. Miorandi, G. S. Canright, and K. Engo-Monsen, "Understanding the spread of epidemics in highly partitioned mobile networks," in BIONETICS '06: Proceedings of the 1st international conference on Bio inspired models of network, information and computing systems. New York, NY, USA: ACM, 2006, p. 2.

[9] S. Sarat and A. Terzis, "On using mobility to propagate malware," in Proceedings of the 5th International Symposium on Modeling and Optimization in Mobile, Ad Hoc, and Wireless Network (WiOpt), April 2007.

[10] M. Bloem, T. Alpcan, and T. Başar, "Optimal and robust epidemic response for multiple networks," in Proc. 46th IEEE Conference on Decision and Control, New Orleans, LA, December 2007. [Online]. Available: papers/cdc07patching.pdf

[11] M. Costa, J. Crowcroft, M. Castro, A. Rowstron, L. Zhou, L. Zhang, and P. Barham, "Vigilante: end-to-end containment of internet worms," in SOSP '05: Proceedings of the twentieth ACM symposium on Operating systems principles. New York, NY, USA: ACM, 2005, pp. 133-147.

[12] M. Lelarge and J. Bolot, "Network externalities and the deployment of security features and protocols in the internet," in SIGMETRICS '08: Proceedings of the 2008 ACM SIGMETRICS international conference on Measurement and modeling of computer systems. New York, NY, USA: ACM, 2008, pp. 37-48.

[13] W. O. Kermack and A. G. McKendrick, "A contribution to the mathematical theory of epidemics," Proceedings of the Royal Society of London. Series A, Containing Papers of a Mathematical and Physical Character, vol. 115, no. 772, pp. 700-721, 1927. [Online]. Available: http://www.jstor.org/stable/94815

[14] D. Fudenberg and D. K. Levine, The Theory of Learning in Games. MIT Press, May 1998.

[15] S. Shahshahani, "A new mathematical framework for the study of linkage and selection," Memoirs of the American Mathematical Society, 1979.

[16] E. Akin, "The differential geometry of population genetics and evolutionary games," in Mathematical and Statistical Developments of Evolutionary Theory, S. Lessard, Ed. Dordrecht: Kluwer, 1990, pp. $1-93$.

[17] D. Monderer and L. Shapley, "Potential games," Games and Economic Behavior, vol. 14, pp. 124-143, 1996.

[18] W. Sandholm, "Evolution and equilibrium under inexact information," Games and Economic Behavior, vol. 44, pp. 343-378, 2003.

[19] T. Roughgarden, Selfish Routing and the Price of Anarchy. Cambridge, Mass.: MIT Press, 2005.

[20] W. Sandholm and R. Lahkar, "The projection dynamic and the geometry of population games," Games and Economic Behavior, 2008.

[21] W. Sandholm, E. Dokumaci, and R. Lahkar, "The projection dynamic and the replicator dynamic," Games and Economic Behavior, 2008.

[22] I. Matei, N. Martins, and J. Baras, "Optimal linear quadratic regulator for markovian jump linear systems, in the presence of one stop-time 
step delayed mode observations," in Proceedings of the 17th IFAC World Congress, Seoul, Korea, July 2008.

[23] — "Almost sure convergence to consensus in markovian random graph," in Proceedings of 2008 IEEE Conference on Decision and Control, December 2008.

[24] — "A consensus problem for markovian random graphs," 2008, submitted for publication.

[25] G. Theodorakopoulos, J. Baras, and J.-Y. Le Boudec, "Dynamic network security deployment under partial information: Global analysis," 2008 , in preparation.

\section{APPENDIX}

We find the equilibrium points $X_{0}, X_{1}, X_{2}$ by solving the system (4) in the three different regions of $I$ specified in the function $p_{S P}: I<I^{*}-\frac{\epsilon}{2}, I^{*}-\frac{\epsilon}{2}<I<I^{*}+\frac{\epsilon}{2}, I>I^{*}+\frac{\epsilon}{2}$. We then verify that the solutions are in the proper region.

For each case, we solve the respective simple system that results:

- $I>I^{*}+\frac{\epsilon}{2}$

The system becomes:

$$
\begin{aligned}
& 0=-2 \beta S I-\gamma S \\
& 0=\left(S-\frac{\delta}{2 \beta}\right) I \\
& 1=S+I+P
\end{aligned}
$$

The only solution is $(S, I, P)=(0,0,1)$, but this contradicts the assumption $I>I^{*}+\frac{\epsilon}{2}$. Note that the solution with $S=\frac{\delta}{2 \beta}$ leads to a negative value for $I$.

- $I<I^{*}-\frac{\epsilon}{2}$

The system becomes:

$$
\begin{aligned}
& 0=-2 \beta S I+\gamma(1-S-I) \\
& 0=\left(S-\frac{\delta}{2 \beta}\right) I \\
& 1=S+I+P
\end{aligned}
$$

This system has the solutions:

$$
\begin{aligned}
& X_{0}=\left(S_{0}, I_{0}, P_{0}\right)=(1,0,0) \\
& X_{1}=\left(S_{1}, I_{1}, P_{1}\right)=\left(\frac{\delta}{2 \beta}, \frac{1-\frac{\delta}{2 \beta}}{1+\frac{\delta}{\gamma}}, \frac{1-\frac{\delta}{2 \beta}}{1+\frac{\gamma}{\delta}}\right)
\end{aligned}
$$

. The second solution, $X_{1}$, is admissible if and only if

$$
\frac{1-\frac{\delta}{2 \beta}}{1+\frac{\delta}{\gamma}}<I^{*}-\frac{\epsilon}{2}
$$

and also

$$
\frac{\delta}{2 \beta} \leq 1
$$

Note that if $\frac{\delta}{2 \beta}=1$, the two points coincide, so we don't need to study $X_{1}$ separately in this case.

We now examine whether $X_{0}$ and $X_{1}$ are (locally) stable equilibrium points. The Jacobian of the system of the first two equations is

$$
J(S, I)=\left(\begin{array}{cc}
-2 \beta I-\gamma & -2 \beta S-\gamma \\
2 \beta I & 2 \beta S-\delta
\end{array}\right)
$$

We evaluate the Jacobian at the point $X_{0}$ :

$$
J\left(X_{0}\right)=J(0,1)=\left(\begin{array}{cc}
-\gamma & -2 \beta-\gamma \\
0 & 2 \beta-\delta
\end{array}\right)
$$

The eigenvalues of $J\left(X_{0}\right)$ are $-\gamma$ and $2 \beta-\delta$. So, $X_{0}$ is stable if and only if $2 \beta<\delta$, in which case note that $X_{1}$ does not exist.

We evaluate the Jacobian at the point $X_{1}$ :

$$
\begin{aligned}
J\left(X_{1}\right) & =J\left(\frac{\delta}{2 \beta}, \frac{1-\frac{\delta}{2 \beta}}{1+\frac{\delta}{\gamma}}\right) \\
& =\left(\begin{array}{cc}
-\frac{2 \beta+\gamma}{1+\frac{\delta}{\gamma}} & -\delta-\gamma \\
\frac{2 \beta-\delta}{1+\frac{\delta}{\gamma}} & 0
\end{array}\right)
\end{aligned}
$$

The eigenvalues of $J\left(X_{1}\right)$ are $\frac{a_{11} \pm \sqrt{a_{11}^{2}+4 a_{12} a_{21}}}{2}$, where $a_{i j}$ are the elements of $J\left(X_{1}\right)\left(a_{22}=0\right)$. Since $a_{11}<0$, the smallest eigenvalue is always negative. The largest one is negative if and only if $a_{12} a_{21}<0 \Leftrightarrow 2 \beta>\delta$. So $X_{1}$ is stable whenever it exists.

If we evaluate the square root $\sqrt{a_{11}^{2}+4 a_{12} a_{21}}$ at the point $\beta=\gamma\left(1+\frac{\gamma}{\delta}\right)^{2}-\frac{\gamma}{2}$, we see that its argument can also take negative values. Since the eigenvalues are a continuous function of $\beta$, they will have an imaginary part for $\beta$ close to $\gamma\left(1+\frac{\gamma}{\delta}\right)^{2}-\frac{\gamma}{2}$, which means that the trajectories spiral towards $X_{1}$.

- $I^{*}-\frac{\epsilon}{2}<I<I^{*}+\frac{\epsilon}{2}$

The system becomes:

$$
\begin{aligned}
0= & -2 \beta S I-\gamma S \frac{1}{\epsilon}\left(I-I^{*}+\frac{\epsilon}{2}\right) \\
& +\gamma(1-S-I)\left(1-\frac{1}{\epsilon}\left(I-I^{*}+\frac{\epsilon}{2}\right)\right) \\
0= & \left(S-\frac{\delta}{2 \beta}\right) I \\
1= & S+I+P
\end{aligned}
$$

In the second equation the only admissible solution is $S=\frac{\delta}{2 \beta}$. For this value of $S$, the first equation gives the following quadratic equation for $I$ :

$I^{2}-\left(\frac{\epsilon}{2}+\frac{\delta \epsilon}{\gamma}+1+I^{*}\right) I+\epsilon\left(1-\frac{\delta}{2 \beta}\right)+I^{*}-\frac{\epsilon}{2}=0$

Defining $f(I)$ to be the quadratic form above, we see that

$$
f\left(I^{*}-\frac{\epsilon}{2}\right)=\epsilon\left(1-\frac{\delta}{2 \beta}-\left(1+\frac{\delta}{\gamma}\right)\left(I^{*}-\frac{\epsilon}{2}\right)\right)
$$

and

$$
f\left(I^{*}+\frac{\epsilon}{2}\right)=-\frac{\epsilon \delta}{\gamma}\left(I^{*}+\frac{\epsilon}{2}+\frac{\gamma}{2 \beta}\right) .
$$

So, $f\left(I^{*}+\frac{\epsilon}{2}\right)$ is always negative, and $f\left(I^{*}-\frac{\epsilon}{2}\right)$ is positive if and only if (11) is not satisfied. That is, a solution for $I_{2}$ exists (and consequently the point $X_{2}$ exists) if and only if $X_{1}$ does not exist. The exact value of $I_{2}$ is given in (10). By evaluating $f\left(I^{*}\right)$ and using (11), we get that $f\left(I^{*}\right)<0$, so actually $I_{2} \in\left(I^{*}-\right.$ $\left.\frac{\epsilon}{2}, I^{*}\right)$.

The Jacobian of the system of the first two equations is $J(S, I)=\left(\begin{array}{cc}-2 \beta I-\gamma & -2 \beta S-\frac{\gamma}{\epsilon}\left(1+I^{*}+\frac{\epsilon}{2}-2 I\right) \\ 2 \beta I & 2 \beta S-\delta\end{array}\right)$ 
We evaluate the Jacobian at the point $X_{2}=\left(\frac{\delta}{2 \beta}, I_{2}\right)$.

$$
J\left(X_{2}\right)=\left(\begin{array}{cc}
-2 \beta I_{2}-\gamma & -\delta-\frac{\gamma}{\epsilon}\left(1+I^{*}+\frac{\epsilon}{2}-2 I_{2}\right) \\
2 \beta I_{2} & 0
\end{array}\right)
$$

The eigenvalues of $J\left(X_{2}\right)$ are $\frac{\alpha_{11} \pm \sqrt{\alpha_{11}^{2}+4 \alpha_{12} \alpha_{21}}}{2}$, where $\alpha_{i j}$ are the elements of $J\left(X_{2}\right)\left(\alpha_{22}=0\right)$. Since $\alpha_{11}<0$, the smallest eigenvalue is always negative. The largest one is negative if and only if $\alpha_{12} \alpha_{21}<0$. But $\alpha_{21}>0$, and $\alpha_{12}=-\delta-\frac{\gamma}{\epsilon}\left(1+I^{*}+\frac{\epsilon}{2}-2 I_{2}\right)$, which is always negative since $I_{2}<1$ and $I_{2}<I^{*}+\frac{\epsilon}{2}$. So the largest eigenvalue is also always negative, and therefore $X_{2}$ is stable whenever it exists.

Since $\lim _{\epsilon \rightarrow 0^{+}} \alpha_{12}=-\infty$, we can see that for small enough values of $\epsilon$ the eigenvalues have an imaginary part, which causes the trajectories to spiral towards $X_{2}$. 